\title{
Microneedling with radiofrequency for improvement in neck skin laxity and texture
}

\begin{abstract}
In recent years, the number of patients presenting with neck rhytides has been increasing. This increase is in part due to the availability of newer non-invasive technology to treat the normal aging process, but also due to the postural and behavioral changes of this generation where people adopt a head down posture in order to be able to use their computers or smartphones. We present the successful treatment of a case of anterior neck skin laxity and texture with microneedling and radiofrequency.
\end{abstract}

Keywords: radiofrequency, microneedling, neck laxity, neck rhytides, biostimulation, collagen remodeling
Volume 4 Issue 2 - 2020

\author{
Mildred Lopez Pineiro, Joely Kaufman \\ Skin Associates of South Florida, USA
}

Correspondence: Joely Kaufman, Skin Associates of South Florida, 4425 Ponce de Leon Blvd, Suite 200

Coral Gables, FL 33I46, USA, Email drjkaufma@gmail.com

Received: February 18, 2020 | Published: March 02, 2020

\section{Introduction}

In recent years, the number of patients presenting with neck rhytides has been increasing. Part of this increase is due to the normal aging process where the skin becomes more lax and redundant, fat accumulates and there is a drop in the underlying anatomical structures. ${ }^{1}$ However, there has also been an increasing younger population coming in for evaluation of anterior neck rhytides. This is thought to be secondary to behavioral and postural changes in this generation where people adopt a head down posture in order to be able to use their computers or smartphones. The main difference is that in this younger population there tends to be deep horizontal neck wrinkles without skin laxity or fat accumulation.

\section{Case report}

We report the case of a 68 year-old white female who came to clinic for evaluation and management of neck skin texture changes and laxity. The patient grew up in South Florida and elicited many episodes of unprotected sun exposure and sunburns. On clinical exam, she had evidence of moderate to severe photodamage with associated rhytides and loss of subcutaneous fat of the anterior neck (Figure 1a). Upon discussion with the patient, it was decided that the best way to accomplish her cosmetic goal was to pursue treatment with the Genius ${ }^{\circledR}$ (Lutronic Aesthetic) microneedling and radiofrequency device. She underwent a series of three treatments 6 weeks apart. Repeat clinical evaluation 6 weeks following her last treatment session showed great improvement in the patient's skin texture, quality and laxity (Figure 1b).

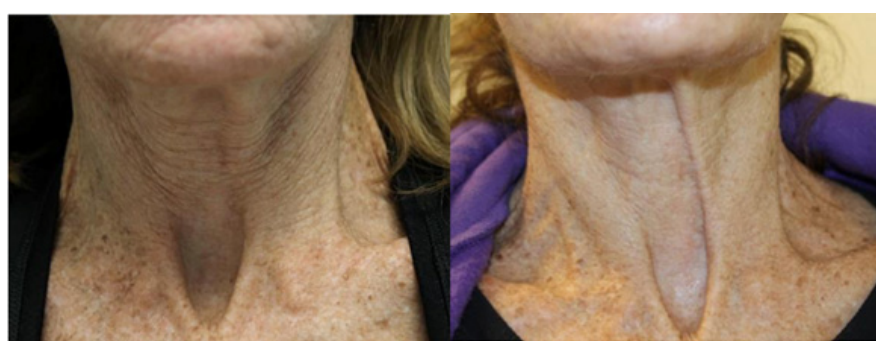

Figure Ia \& Ib Before and after three Genius treatment sessions of a 68 year-old female's anterior neck.

\section{Discussion}

One of the newer reported preferred methods for improving neck skin with the use of non-ablative technology is intradermal radiofrequency with microneedling. ${ }^{1,2}$ Radiofrequency has been used in the medical field for decades, and since the early 2000s has been reported in the dermatologic literature for its use in cosmetic procedures. Radiofrequency delivers heat into the target tissue, which generates an electrothermal reaction that achieves controlled coagulation of the tissue. This causes collagen contraction along with a wound healing response, which then cascades into collagen remodeling and skin tightening. ${ }^{3}$

The favored device in our clinical practice is the FDA cleared Genius $^{\circledR}$ (Lutronic Aesthetic). This device consists of a 49 pin tip that is $1 \mathrm{~cm} \times 1 \mathrm{~cm}$ in size and uses a bipolar array of insulated microneedles to deliver heat to target tissues. The handpiece is placed with moderate to firm pressure on the skin, the needle array is automatically inserted into the skin and radiofrequency is delivered, then the needles automatically retract. The permeability of topical agents is transiently increased and therefore, this treatment can be combined with other procedures such as application of platelet rich plasma. Ideally treatments are spaced 4-6 weeks apart with best results achieved after 2 to 4 treatments. It is contraindicated in patients with compromised wound healing, pregnancy/breastfeeding, skin allergy to topical medications, history of keloids or active skin disorders with pathergy and/or history of implanted medical devices.

Each treatment typically consists of three passes that deliver energy starting in the deep dermis and subsequently more superficially in the dermal skin. Per Chopra et al., ${ }^{4}$ the average anterior neck dermal thickness is $1.3 \mathrm{~mm}$ with a standard deviation of $0.5 \mathrm{~mm} .{ }^{4}$ Therefore, for our anterior neck treatments we utilize settings that range from $1.0 \mathrm{~mm}$ to $2.0 \mathrm{~mm}$ in needle depth with a needle energy ranging from 14 to $20 \mathrm{~mJ} / \mathrm{pin}$. The specific settings applied depend on the patient's age and skin thickness, photodamage, Fitzpatrick skin type as well as preferred patient downtime. The most commonly reported patient complaint is procedure related pain, which is tolerable and typically well controlled with the use of topical anesthesia. Self-resolving mild erythema, edema and bruising may also occur. Downtime of 2 to 4 days is typical and expected. 


\section{Conclusion}

As with any non-invasive biostimulatory cosmetic procedure, it can take 6 to 8 weeks to see the final cosmetic results from the series of treatments. However, the results are very promising (Figure 2a) (Figure $2 b$ ). We believe micro needling with radiofrequency provides a precise and controlled technique for achieving improved skin laxity, texture and redundancy not only for the anterior neck, but also for the face and body. With the majority of cosmetic patients now seeking non-invasive modalities to improve the appearance of their skin and body, it is important for providers to be aware of the different uses each clinical device can potentially offer. More clinical studies are necessary in order to standardize the practice and clinical use of this technology.

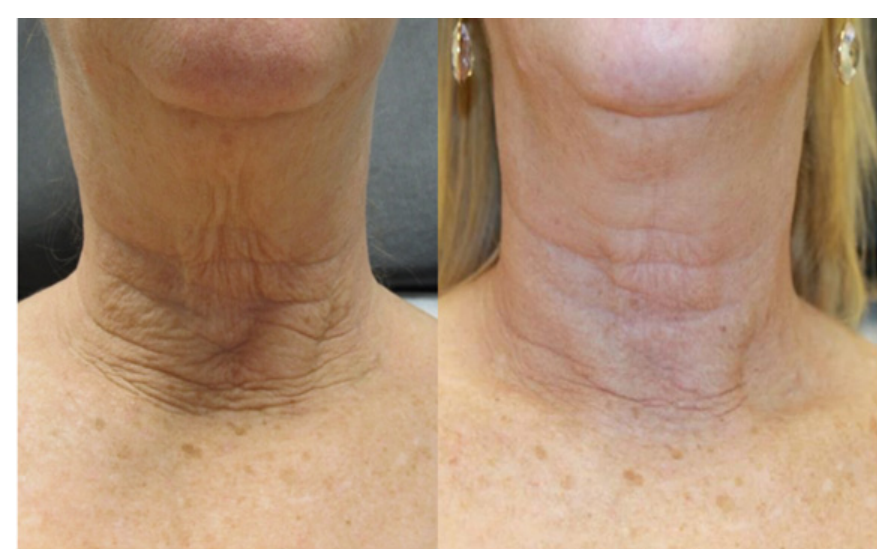

Figure 2a \& 2b Before and after three Genius treatment sessions of a 62 year-old female's anterior neck.

\section{Conflicts of interest}

The author declares that there is no conflicts of interests.

\section{Acknowledgements}

None.

\section{Funding}

None.

\section{References}

1. Hsu TS, Kaminer MS. The use of nonablative radiofrequency technology to tighten the lower face and neck. Semin Cutan Med Surg. 2003;22:115123

2. Alster TS, Tanzi E. Improvement of neck and cheek laxity with a nonablative radiofrequency device: a lifting experience. Dermatol Surg. 2004;30:503-507.

3. Hyun MY, Li K, Kim BJ, et al. Novel treatment of neck wrinkles with an intradermal radiofrequency device. Ann Dermatol. 2015;27(1):79-81.

4. Chopra K, Calva D, Sosin M, et al. A comprehensive examination of topographic thickness of skin in the human face. Aesthet Surg J. 2015;35(8):1007-1013. 